Einführung in das Schwerpunktthema

\title{
Unternehmen auf dem Weg zur Nachhaltigkeit
}

Von Thomas Loew er Brundtlandbericht sowie die Rio-Konferenz vor acht Jahren markieren den Beginn der Diskussion um nachhaltige Entwicklung in Wissenschaft und Politik. Weniger als die Hälfte der Unternehmen hat sich jedoch bis heute mit der Thematik auf Geschäftsführungsebene beschäftigt. Und bei etwa einem Viertel der im Rahmen einer gemeinsamen Studie des IÖW und des Münchener ifo-Instituts (1) befragten Managementvertreter war der Begriff noch gänzlich sind daher weitgehend bekannt. Für das Umweltmanagement stehen zahlreiche Controllinginstrumente zur Verfügung und bereits seit mehreren Jahren können Unternehmen ihre Umweltmanagementsysteme nach dem EG-Öko-Audit-System (EMAS) oder der internationalen Norm ISO 14.001 validieren bzw. zertifizieren lassen. Wichtige Erfolge sind inzwischen auf freiwilliger Basis oder aufgrund der Umweltgesetzgebung beim produktionsbezogenen Umweltschutz erreicht worden. Die großen Herausforderungen werden

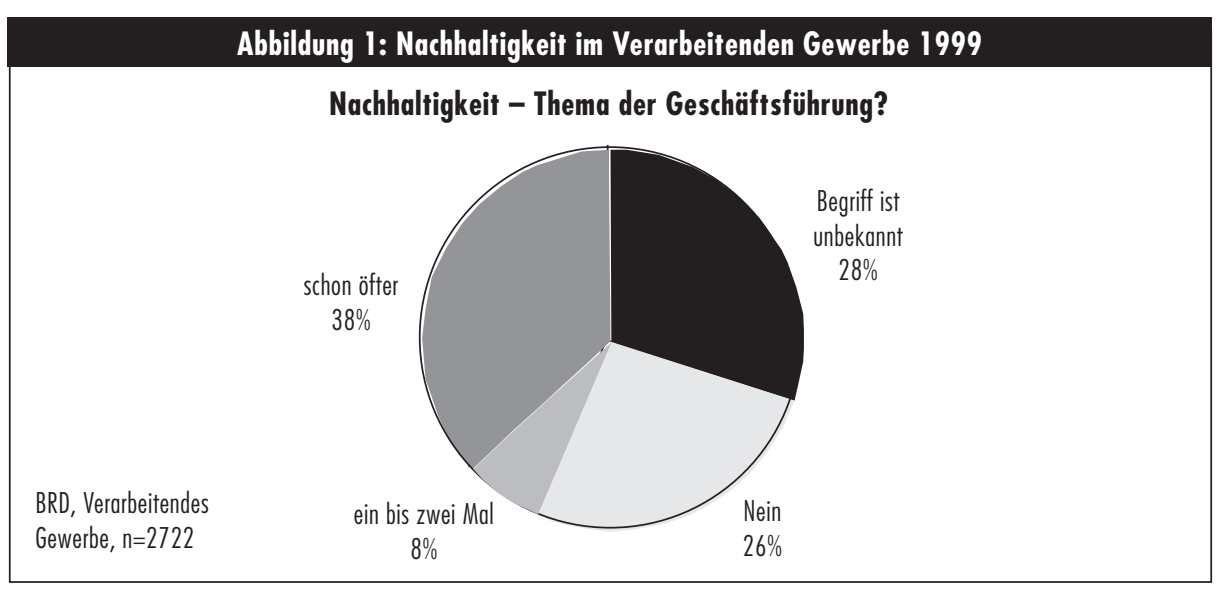

Quelle: Siehe Anmerkung (1)

unbekannt (siehe Abbildung 1).

Die Debatte um Nachhaltigkeit von Unternehmen leidet unter der Komplexität und der teilweisen Widersprüchlichkeit der Anforderungen, die damit an die Unternehmen gestellt werden. So ist wenig verwunderlich, dass es bislang überwiegend große Unternehmen sind, die sich mit Nachhaltigkeit auseinander setzen. Hier finden bereits interessante Entwicklungen statt. Erste Vorreiter haben bereits ihre Umweltberichterstattung in einer Nachhaltigkeitsberichterstattung aufgehen lassen und zeigen dort, wie sie die Anforderungen in der ökologischen, ökonomischen und sozialen Dimension der Nachhaltigkeit interpretieren.

Die Unterscheidung dieser drei Dimensionen ist hilfreich, um den Diskussions- und Umsetzungsstand in den Unternehmen beschreiben zu können. Die Nachhaltigkeitsdiskussion hat sich in den Unternehmen, wie übrigens auch analog im IÖW, aus dem Umweltthema entwickelt. Die Herausforderungen der ökologischen Dimension heute in der Produktentwicklung gesehen, wo es gilt, die Umweltauswirkungen über den gesamten Produktlebenszyklus drastisch zu reduzieren. Die wesentliche Anforderung besteht darin, dass in Zukunft nicht nur die Ökoeffizienz gesteigert wird, sondern auch die absoluten Umwelteinwirkungen reduziert werden.

\section{Interpretation von ökonomischer Nachhaltigkeit umstritten}

Die ökonomische Dimension wird oftmals mit der Wirtschaftlichkeit des Unternehmens gleich gesetzt. In diesem Sinne wird Nachhaltigkeit auch von konservativen Unternehmens- und Verbandsvertretern gerne aufgegriffen: Umweltschutzanforderungen an Unternehmen müssten sich am wirtschaftlich Machbaren orientieren. Diese Interpretation trifft jedoch nicht die Problemlagen, die ursprünglich mit ökonomischer Nachhaltigkeit gemeint waren. Die ökonomische Dimension wurde und sollte auch weiterhin primär als volks- wirtschaftliche Nachhaltigkeit interpretiert werden. So ist es in diesem Kontext zwar grundsätzlich richtig, dass für eine gesunde Volkswirtschaft eine große Anzahl einzelwirtschaftlich erfolgreicher Unternehmen erforderlich ist, aber gleichzeitig können ökologische oder soziale Gründe durchaus einen Strukturwandel erfordern, der die Schließung von einzelnen Unternehmen bedeuten kann.

Aus der volkswirtschaftlichen Sicht betreffen die Anforderungen und der Beitrag der Unternehmen nicht nur den erwirtschafteten Gewinn, sondern insbesondere auch Fragen zur Verteilung der Wertschöpfung. Welche Anforderungen hier an Unternehmen, angefangen bei den kleinen und national agierenden bis hin zu den Global Players zu stellen sind, ist bislang noch wenig erforscht. In der Diskussion um Nachhaltigkeitsberichterstattung (vgl. auch den Beitrag von Raupach/ Clausen in diesem Heft), dokumentiert in dem Leitfaden der Global Reporting Initiative (2), werden verschiedene Kennzahlen vorgeschlagen, mit denen die Unternehmen über ihren volkswirtschaftlichen Beitrag berichten sollen. Dabei bleibt aber völlig offen, welche Zielwerte oder -korridore diese Kennzahlen erreichen sollen.

\section{Kontroverse soziale Dimension}

Wiederum anders stellt sich die Situation in der sozialen Dimension dar. Hier versammeln sich vielfältige Anforderungen aus sehr unterschiedlichen Richtungen, die zum Teil schon deutlich ältere Wurzeln haben als die Umweltschutzdebatte. Hierzu gehören Fragen der Mitbestimmung, Entlohnung, Qualifizierung, Arbeitszeiten sowie soziale Aspekte bei der Beschaffung (Stichwort Kinderarbeit), um nur einige zu nennen (vgl. auch den Beitrag von Lohrie). Hier stellt sich also die Aufgabe, die Vielfalt zu strukturieren und Prioritäten zu setzen. Auch bei der sozialen Nachhaltigkeit neigen die Unternehmen dazu, primär vor ihrer eigenen Haustïr zu kehren, das heißt im Mittelpunkt der Aufmerksamkeit stehen soziale Fragestellungen in Bezug auf die Mitarbeiter. Die konsequente Analyse ihrer Produkte auf soziale Nachhaltigkeit steht dagegen noch aus.

Während die Unterscheidung der Dimensionen für übergreifende Betrachtungen hilfreich ist, kann sie bei der Zuordnung von Anforderungen in der Praxis auch hinderlich sein. Betrachtet man beispielsweise die Entlohnung der Mitarbeiter dann ist unklar, ob es sich hier um einen Aspekt sozialer oder ökonomischer Nachhaltigkeit 
handelt. Für die Beteiligten, also die Unternehmen, die Mitarbeiter und deren Interessenvertreter ist diese Fragestellung letztendlich unwichtig. Für die Erstellung von Visionen und Modellen nachhaltiger Unternehmen oder für die Beurteilung der Nachhaltigkeit einzelner Unternehmen, beispielsweise im Kontext ökologisch-ethischer Finanzanlagen sollten diese Zuordnungsfragen aber noch geklärt werden. Als Lösung dürften hier vielfach nur Konventionen in Frage kommen.

\section{- Unvereinbare Wege?}

Immer wieder wird betont, dass nachhaltige Entwicklung eher als Prozess denn als Ziel anzusehen ist. Damit wird der Unsicherheit und insbesondere auch den Konflikten über die Zielrichtung aus dem Weg gegangen. Derzeit lassen sich zwei gegensätzliche Richtungen unterscheiden. Die eine versucht eine nachhaltige Entwicklung durch eine radikale Steigerung der Ökoeffizienz zu erreichen. Ergänzend wird versucht die sozialen Anforderungen, basierend auf den christlichen Werten des Abendlands, zusätzlich zu berücksichtigen und auch hier wird zunächst nach den win-win-Situationen gesucht. Der Idealfall liegt demnach in triple-win-Lösungen (vgl. auch den Beitrag von von Gleich et al.), die in der Realität wohl nur selten anzutreffen sind. Diese „ÖkoEffizienz-Nachhaltigkeit" bedeutet keine Änderung unserer Wirtschaftsweise und wird daher auch von der Wirtschaft zunehmend aufgegriffen.

Dem gegenüber steht eine ,Weniger ist mehr“Nachhaltigkeit, nach der Stoffströme, Konsum und Arbeitszeit reduziert werden sollen (vgl. den Beitrag von Flieger und Sing). So erhalten die Erwerbstätigen mehr Freizeit, können ein erfuillteres Leben mit besseren sozialen Kontakten führen. Konsum als Kompensation für den Arbeitsstress verliert an Bedeutung. Aus der Vogelperspektive, mit dem Blick auf die sozialen und ökologischen Problemlagen, erscheint diese Richtung plausibler. Ungeklärt ist die wirtschaftliche Machbarkeit und insbesondere die Umsetzung, denn zweifelsohne beinhaltet dieser Weg eine bedeutsame Änderung unserer bisherigen Form des Wirtschaftens. Nicht Gewinn und Wachstum, sondern Suffizienz stehen im Vordergrund. Offensichtlich kein attraktiver Vorschlag für Unternehmen und die Mehrheit unserer Gesellschaft.

\section{- Die Beiträge im Überblick}

Im vorliegenden Schwerpunktthema kommen Wirtschaft und Wissenschaft gleichermaßen zu Wort. Damit soll, ganz im Sinne der Forderungen zahlreicher Studien, der Dialog über das Leitbild Nachhaltigkeit weiter geführt werden. Eine neue, und in den kommenden Jahren sicherlich wichtige, Stimme der Wirtschaft in diesem Dialogprozess ist das Forum für Nachhaltigkeit der deutschen Wirtschaft. Es wurde im Sommer 2000 von 17 Großunternehmen gegründet und soll als Denkfabrik neue Ideen sammeln, entwickeln und aktiv in die politische Diskussion einbringen. Das Forum wird von Wolf-Eberhard Schiegl und Ralph Thurm, Siemens AG, näher vorgestellt.

Welche unterschiedlichen Facetten die Erweiterung des Blickwinkels unter dem Stichwort Nachhaltigkeit in der Praxis aufweisen kann, zeigen die Beiträge aus zwei Großunternehmen. Achim Lohrie beschreibt, wie der OTTO-Versand den Sozialstandard SA 8000 nutzt, um (nicht nur) bei der Beschaffung soziale Aspekte zu berücksichtigen. Ignacio Campino berichtet über die Chancen und Risiken bei der Schlüsselressource Mitarbeiter im Wandlungsprozess der Deutschen Telekom zu einem Global Player. Zugleich beleuchtet er die Herausforderung, die ökologische und soziale Dimension mit den ökonomischen Wettbewerbsbedingungen in Einklang zu bringen.

Aus wissenschaftlicher Sicht werfen sich eine Reihe von zum Teil sehr weitreichenden Fragen auf. Warum befassen sich Unternehmen überhaupt mit dem Thema, wo bestehen Anknüpfungspunkte, die von der Politik und den Anspruchsgruppen genutzt werden können? Arnim von Gleich, Manuel Gottschick und DirkJepsen unterscheiden hier moralische Überzeugungen, die Realisierung von Kostenvorteilen und langfristige Marktstrategien. Dabei räumen sie aufgrund von Veränderungen auf den Märkten letzteren eine zunehmende Bedeutung ein.

Jens Clausen und Volker Stablmann fokussieren auf die ökologische Dimension und zeigen die ungelösten Probleme bei der Bestimmung der Umweltleistung unter Nachhaltigkeitsgesichtspunkten auf. Obgleich es inzwischen eine ISO Norm für die Umweltleistungsbewertung gibt, ist weitgehend offen, wie jenseits von Verbesserungen der Öko-Effizienz die gesamten Umweltwirkungen eines Unternehmens zu beurteilen sind. In den Unternehmen herrscht vielfach noch Unklarheit, welche sozialen Anforderungen aus der Nachhaltigkeitsdebatte zu priorisieren sind. Hier bietet es sich für einen organisierten Suchprozess an, das im Umwelt- und Qualitätsmanagement bewährte Audit-Prinzip auf Nachhaltigkeit anzuwenden. Welche Ergebnisse ein derartiges Nachhaltigkeits-Audit gerade bei den sozialen Fragestellungen haben kann, zeigen Alexandra Lux, Claudia Empacher und Thomas Kluge am Praxisbeispiel eines Finanzdienstleisters.

Auch Burghard Flieger und Elmar Sing thematisieren soziale Nachhaltigkeit. Sie zeigen mit ihren Überlegungen zur Nachhaltigkeit von Produkten, wo die großen win-win-Potenziale zwischen der ökologischen und sozialen Dimension liegen: im „weniger ist mehr“. Für die erforderliche sozialökologische Produktentwicklung können die Unternehmen u.a. auf den Sachverstand der NGOs zugreifen - wenn sie die geeigneten Dialogformen wählen.

Eine andere Form des Dialogs wird mit den immer zahlreicher publizierten Nachhaltigkeitsberichten geführt. Sie sind zugleich ein Instrument, mit dem die Unternehmen ihren eigenen Entwicklungsprozess vorantreiben können. Den derzeitigen Entwicklungsstand sowie Perspektiven gerade auch für kleinere Unternehmen beschreiben Michaela Raupach und Jens Clausen.

Es hat seit Rio fast ein Jahrzehnt gedauert, bis die Wirtschaft und die sozial-ökologische Wirtschaftsforschung begonnen haben, sich ernsthaft mit Ausgestaltungsfragen des Leitbilds nachhaltiger Entwicklung auseinander zu setzen. Inzwischen sind aber die erforderlichen Strukturen ausgebildet, so dass die Arbeiten und Diskussionen nun deutlich an Fahrt gewinnen werden.

\section{Anmerkungen}

(1) Vgl. Sprenger, R.U./ Petschow, U./ Fichter, K./ Loew, T./ dos Matos, I./ Wackerbauer, J.: Standortbedingungen Berlins für umwelttechnische Produktionen und Dienstleistungen und für eine nachhaltige Entwicklung der Wirtschaft, Schriftenreihe des IÖW 155/01, Berlin 2001 (im Erscheinen). Das Forschungsvorhaben wurde von der Berliner Senatsverwaltung für Stadtentwicklung gefördert.

(2) Vgl. Global Reporting Initiative: Sustainability Reporting Guidelines. Boston, June 2000.

\section{Der Autor}

Thomas Loew ist wissenschaftlicher Mitarbeiter im IÖW-Forschungsfeld Ökologische Unternehmenspolitik. Kontakt: Institut für ökologische Wirtschaftsforschung, Potsdamer Str.105, 10785 Berlin. Tel. 030/ 884594-0, Fax 030/ 8825439, E-mail: Thomas.Loew@ioew.de 
(c) 20I0 Authors; licensee IÖW and oekom verlag. This is an article distributed under the terms of the Creative Commons Attribution Non-Commercial No Derivates License (http://creativecommons.org/licenses/by-nc-nd/3.o/), which permits unrestricted use, distribution, and reproduction in any medium, provided the original work is properly cited. 